$\underline{\text { Reports }}$

\title{
Where there was no COVID-19: strengthening health systems response to disease threats in Tabora, Tanzania
}

\author{
Shegufta S Sikder ${ }^{1}$, Amani Idfonce ${ }^{2}$ \\ ${ }^{1}$ CARE USA, Washington DC, USA, ${ }^{2}$ CARE International, Tabora, Tanzania \\ Keywords: gender-based violence, gender integration, covid-19, health systems \\ https://doi.org/10.29392/001c.31594
}

\section{Journal of Global Health Reports}

Vol. 6, 2022

\section{Background}

In May 2020, the international non-governmental organization Cooperative for Assistance and Relief Everywhere (CARE) sought to enable health systems in Tanzania to address COVID-19 health response. CARE leveraged existing partnerships with the Ministry of Health, Community Development, Gender, Elderly and Children and the President's Office of Regional Administration and Local Government in the largest geographic region of Tanzania, Tabora Region. At the time, the government declared Tanzania to be free from COVID-19 and did not permit partners to implement COVID-19 programming. In this context, CARE implemented integrated health systems strengthening support for government response to not only COVID-19 but also other infectious diseases.

\section{Methods}

We describe program implementation using monitoring reports generated by CARE colleagues to inform planning of integrated health programs that are gender-responsive. The initiative supported regional and local capacity building on infection prevention and control, household hygiene, infectious disease surveillance, and response to gender-based violence according to Tanzania's national guidelines. These efforts utilized existing platforms and workers, including health care workers, community health workers, community groups, and government health management teams at the regional and district levels.

\section{Results}

CARE supported Tabora Region to develop 20 regional trainers who provide on-site mentorship and supportive supervision on infection prevention and control to health care workers. Nearly all (93\%) trained community members on Violence Against Women and Children (VAWC) Committees expressed confidence in response to Gender-Based Violence in accordance with Tanzania's National VAWC Action Plan. Trained Community Health Workers continue to link communities to health information and services.

\section{Conclusions}

Implementation of a holistic health system strengthening approach with a gender lens positioned Tabora Region for response to infectious disease threats including COVID-19. Program managers seeking to address COVID-19 in their contexts should strengthen health systems at regional and district levels as well as community structures to enable resilient response to disease threats including but not limited to COVID-19.

The first reported case of COVID-19 in Tanzania was on March 16, 2020. ${ }^{1}$ While data on COVID-19 cases were originally reported through the national Ministry of Health, Community Development, Gender, Elderly and Children (MoHCDGEC), the previous government stopped reporting data on COVID-19 cases in May 2020. ${ }^{2}$ The former government declared Tanzania to be "COVID-free" in June 2020. ${ }^{3}$ In March 2021, government leadership changed with the induction of President Samia Hassan following demise of the prior President John Magufuli. Under President Hassan's government, Tanzania resumed reporting COVID-19 cases in July 2021, revised the country's National COVID-19 Response Plan, and initiated COVID-19 vaccinations in country through COVID-19 Vaccines Global Access, or COVAX, initiative. ${ }^{4}$ 
In May 2020, prior to the change in Tanzania's government, the International Non-Governmental Organization (INGO) known as Cooperative for Assistance and Relief Everywhere (CARE) received funding from Bloomberg Philanthropies for COVID-19 response. Given strong existing partnerships with MoHCDGEC as well the President's Office for Regional Administration and Local Government (PORALG), CARE identified the Tabora Region of Tanzania as the location to implement COVID-19 response building on government collaboration. Tabora is the largest geographic region in Tanzania and has among some of the most remote and underserved districts in the country. ${ }^{5}$

\section{PROGRAM DESCRIPTION}

This funding leveraged an existing four-year initiative in Tabora Region funded by the Government of Canada to improve gender-equitable access and quality of reproductive, maternal and newborn health services across health facilities and communities (known as the Tabora Maternal and Newborn Health Initiative, or TAMANI. ${ }^{6}$ ) We sought to extend this partnership to enhance ability of health systems to respond to integrated disease threats including COVID-19 in all levels of health care in Tabora Region of Tanzania. We built on strategic opportunities to leverage the government's recognition of the need for public health preparedness through its adoption of the Integrated Disease Surveillance and Response (IDSR) system for all disease surveillance activities in the country in $2019 .{ }^{7}$

As an organization, CARE prioritizes gender equality aligned with Sustainable Development Goal $5 .^{8}$ In order to identify how to address gender during crises, CARE implements a tool known as rapid gender analysis. ${ }^{9}$ During the initial onset of COVID-19 in 2020, CARE implemented a Rapid Gender Analysis to understand the impacts of COVID-19 on communities across more than 50 countries, including Tanzania. ${ }^{10}$ These analyses highlighted the gendered impacts of the pandemic, particularly increases in gender-based violence (GBV), harassment and restrictions on women's access to resources and decision-making. ${ }^{10,11}$ In Tanzania, CARE collaborated with digital partner Viamo to conduct a mobile survey via interactive voice response. A sample of 10,000 known phone numbers of beneficiaries from eight projects, including TAMANI were contacted, with 1,177 complete responses. Both men and women, 54\% and $56 \%$ respectively, reported an increase of harassment and violence during the pandemic at the time of survey collection. ${ }^{6}$ Survey results also revealed that while women reported the ability to report cases of GBV, a lower proportion of women were willing to report cases in the COVID-19 context. ${ }^{6}$ Given CARE's focus on advancing gender equality and the surveys showed the dire gender impacts of the pandemic, CARE sought to integrate a gender-responsive approach.

\section{METHODS}

We describe program implementation using quarterly monitoring reports generated by CARE Tanzania colleagues. We

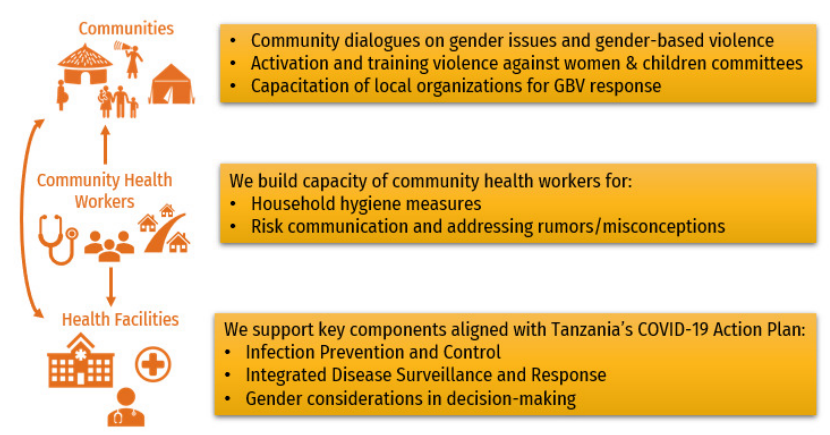

\section{Figure 1. Intervention components by platform in Tabora, Tanzania}

then describe key achievements from quarterly program monitoring data collected between June 2020 to August 2021 as well as from a digital survey conducted by an external partner, Viamo. CARE built on partnerships with government Regional and Council/District Health Management Teams and existing community groups for integrated health systems response to disease threats in Tabora Region.

\section{OVERVIEW OF APPROACH AND RESPONSE}

The funding from Bloomberg Philanthropies complemented by the TAMANI project supported 23 staff for implementation of activities from May 2020 to August 2021 in all eight districts of the region. CARE staff in Tabora Region included one senior manager, five district managers, one gender manager, one monitoring and evaluation manager, seven project officers who were responsible for community mobilization, one accountant, one administrative staff member, and six drivers. Figure 1 presents an overview of intervention components. Community health workers are essential linkages connecting communities to health facilities. Interventions across community, CHW, and health facility platforms comprised our integrated health systems response to disease threats.

CARE supported the Tabora Region to generate mentors for Infection Prevention and Control (IPC) and capacity in Integrated Disease Surveillance and Response (IDSR) to operationalize existing national guidelines. For health care worker capacity building on IDSR, the government's regional medical office identified health facilities in the region with poor performance regarding timely reporting and data quality of IDSR indicators in the District Health Information System (DHIS-2). DHIS-2 is used across Tanzania for tracking data on health interventions from primary to tertiary levels of health care. Based on this list of identified health facilities, the District Medical offices selected health care workers from those facilities to participate in the trainings. As the region has eight districts, five health care workers (including nurses, doctors, and midwives) were identified to represent each district for a total of 40 trainees. A different set of 40 health care workers were identified for trainings on IPC and GBV response from health facilities that provide comprehensive emergency obstetric and newborn care and with high load of GBV cases, respectively. The project also supported capacity building to Community 
Health Workers (CHWs) for awareness creation on household hygiene and reporting on key indicators.

CARE leveraged existing community platforms for GBV response. Within existing community savings groups, CARE helped to facilitate dialogues to enable participants to discuss gender and social determinants of health and action plans to address challenges. CARE also built capacity among local organizations for GBV response in coordination with government authorities, in alignment with the National Action Plan to End Violence Against Women and Children (NPA-VAWC). ${ }^{12}$ Furthermore, CARE supported the formation of Violence Against Women and Children Committees at the village level and provided them with capacity building for GBV response.

\section{PLATFORMS FOR HEALTH SYSTEMS STRENGTHENING}

\section{HEALTH FACILITIES AT REGIONAL, DISTRICT, AND LOCAL LEVELS}

CARE worked with MoHCDGEC to implement capacity building to Regional and Council/District Health Management Teams on Integrated Disease Surveillance and Response in health care settings. First, CARE's project manager worked with three national government trainers to train 54 district health managers (17 female, 37 male) on key components of disease surveillance per Tanzania's IDSR Guidelines. ${ }^{13}$ Key components included case identification, recording and reporting; data analysis and interpretation; response for outbreaks and other public health importance events; and risk communication and community engagement. The three national government trainers led this training with logistics and facilitation support from CARE's project manager. Four national facilitators trained 40 health care workers ( 22 female, 18 male) on government guidelines on disease surveillance at facility and community levels. Health facility surveillance covered case identification and the use of digital tools in reporting. Community-based surveillance covered rumors management and the use of CHWs in surveillance at the community. In this cascade training approach, CARE leveraged existing national trainers to build regionally capacitated trainers on IDSR.

Regional government officers from community development and social welfare conducted training with logistics and facilitation support from CARE to 32 district health managers (18 female, 14 male) on consideration of gender issues in planning and decision making. The purpose was to build awareness among health managers on how women and girls are prone to gender inequalities and how these can be addressed. The training covered approaches of reaching the needs of women and girls and addressing needs in planning and budgeting. ${ }^{14}$ (In Tanzania, Comprehensive Council Health Plans are coordinated by district councils annually to plan and budget for the coming year's essential health and social welfare interventions.) CARE's gender manager worked with regional community development and social welfare departments to capacitate 40 health care workers ( 23 female, 17 male) for response to GBV issues including those accelerated by COVID-19. The

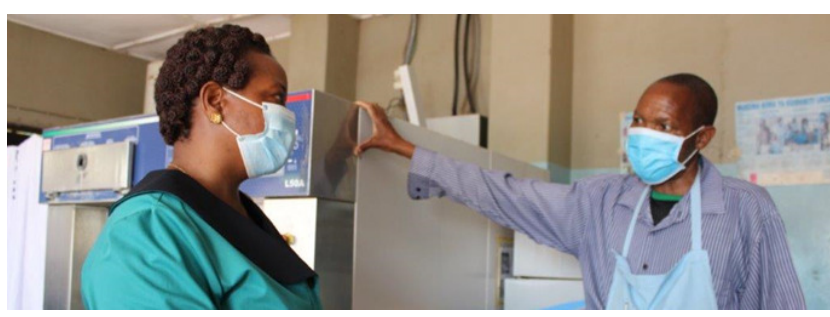

Photo A. A regional mentor providing guidance on
infection prevention and control at Nzega Town Hospital in Nzega Town Council, Tabora Region (CARE, 2021)

training built on Tanzania's National Gender and Respectful Maternity Care Guideline and National Plan for Action to End Violence Against Women and Children. ${ }^{12}$

CARE supported Tabora Region to generate 20 regional infection prevention control (IPC) trainers/mentors (11 female, nine male) to build regional and local capacity. These mentors were identified from existing health care workers and regional/district health managers. Six trained regional IPC mentors facilitated IPC training under supervision of national facilitators to 40 health care workers (23 female, 17 male) in the region. The training content focused on Tanzania's National IPC Guideline, with key components including avoiding health-care-associated infections and how to handle viral infections. The trained regional mentors together with five national facilitators under the project support conducted on-site mentorship and supportive supervision on IPC to 248 (134 female, 114 male) health care workers (doctors, nurses and midwives) throughout Tabora Region. As of now, the region has capacity to conduct regular supportive supervision visits by using its own regional mentors instead of inviting national mentors from the ministry. Across integrated disease surveillance and infection prevention and control, CARE connected existing national trainers to build regional capacity for response across disease threats.

Through discussion with regional and council/district health authorities on areas of support needed, CARE procured 216 mobile foot-operated hand washing stations for use in government health facilities. These handwashing stations are expected to strengthen hand hygiene in health care settings. This support addressed Tanzania's National Guideline for Water Sanitation and Hygiene in Health Care Facilities. ${ }^{11}$

\section{COMMUNITY HEALTH WORKERS}

In response to COVID-19, CARE assisted the Tabora Region in training CHWs on household hygiene. Hygiene practices are recognized as one of the ways that households can mitigate the spread of infectious diseases. ${ }^{12}$ District Health Officers under regional support trained a total of $446 \mathrm{CHWs}$ (238 female, 208 male) from all eight district councils. CARE facilitated logistics and facilitation on roles of CHWs, building on CARE's previous program support to recruit 1,000 CHWs as part of the TAMANI project. The areas covered during the training included environment and waste 
management; basic standards of hygiene at household level, safe water, toilet hygiene, handwashing, waste management and utensil hygiene; control of infectious diseases and key preventive measures in infection control of COVID-19, cholera, diarrhea, etc.; and strengthening resilience during outbreaks of infectious diseases.

\section{COMMUNITY DIALOGUES}

Within existing Village Savings and Loan Associations, CARE facilitated discussions on the gendered impacts of COVID-19. CARE trained community facilitators, who led sessions under supervision of district officials and project staff. These dialogues allowed participants to identify action plans to address gender relations in the COVID-19 context. Group dialogues and community engagement activities were conducted in smaller groups to align with safety guidelines.

Facilitators followed the community action cycle of CARE's Social Analysis and Action (SAA) approach, ${ }^{15}$ which engages participants to address gender imbalances through actions in their personal lives. Facilitators supported community groups to identify gender inequality issues accelerated by COVID-19 and set action items to address those issues. SAA dialogues addressed key gender issues raised by CARE's Rapid Gender Analysis ${ }^{11}$ and United Nations Development Programme. ${ }^{16}$ The dialogues engaged 852 participants (516 female, 336 male) across 24 communities in Tabora Region.

\section{BUILDING LOCAL CAPACITY FOR GBV RESPONSE}

At project onset, CARE conducted a mapping to identify local organizations working on GBV in the area. Based on this mapping CARE trained 18 local organizations on how to integrate GBV responses in their programming at the community level. The training was co-facilitated by CARE, MoHCDGEC, Gender and Children Desk officers and Regional Community Development and Social Welfare departments. Topics covered gender concepts, NPA-VAWC, GBV response and safety planning, and socioeconomic issues related to GBV in the context of COVID-19. CARE shared findings from the community dialogues as part of demonstrating gender implications of the pandemic. Each organization developed an action plan to address GBV in their localities. Action plans included awareness creation, legal aid to victims, linkage for further services etc., key components of Tanzania's NPA-VAWC.

CARE worked with the Regional Community Development Department and Social Welfare Unit to support formulation of village committees for ending violence against women and children as well as capacity building. This effort built on NPA-VAWC, which requires each village to have a functional committee. A total of 32 committees (four villages per district) were formulated and trained on NPAVAWC strategy. The 744 training participants (358 female, 386 male) represented various groups: government officials, religious leaders, disabled, elders, influential people, children, etc. The training covered various topics including gender concepts; reporting mechanisms; GBV and norms/

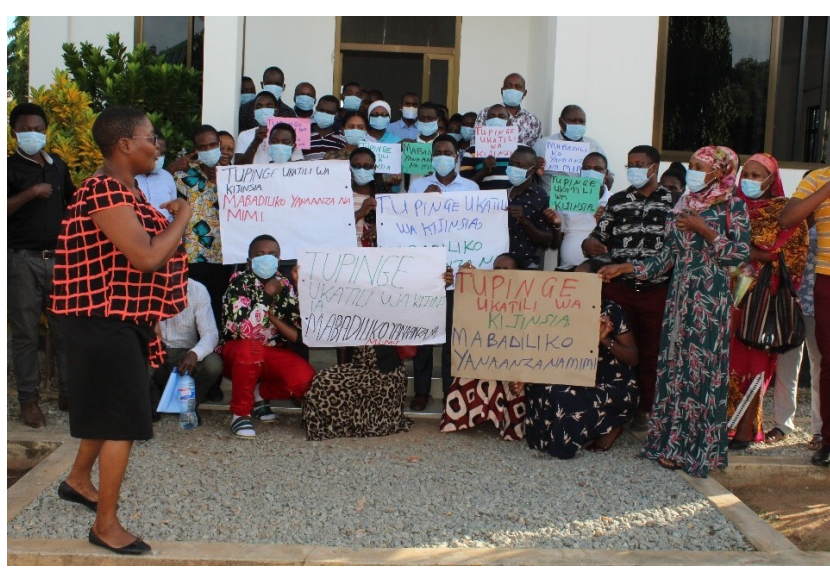

Photo B. Health care workers and regional and district government officers trained in GBV response with signs reading "Let's fight against GBV, it begins with me" (CARE, 2021)

values; roles of the committees; and thematic areas of NPAVAWC.

\section{RESULTS}

\section{KEY ACHIEVEMENTS}

CARE collaborated with digital partner Viamo to conduct a mobile survey in June 2021 among participants of community dialogues/ SAA and VAWC committees. Out of 755 participants called, 421 (56\%) completed the survey on their mobile phones (199 community dialogues \& 222 VAWC participants). Nearly all respondents $(89 \%, n=177)$ have had follow up discussions in their community groups on the gender actions established in community dialogues. Nearly all respondents $(93 \%, n=206)$ expressed confidence to respond to GBV cases following the VAWC training, with the majority developing plans to respond to GBV.

Prior to these activities, Tabora Region lacked trained government mentors on IPC. As of August 2021, the region has internal capacity to conduct IPC mentorship by using 20 trained regional mentors for regular and sustained IPC mentorship. Surveys administered to regional and district management teams showed high average scores of abilities in risk communication and emergency preparedness as well as surveillance. ${ }^{6}$ In the words of IPC mentor Pandu Revocatus Daud, "IPC training has helped me to transform my knowledge from old understanding to new ones as per updated National Guideline of 2018 which are essential in infection control."

The National Sanitation Management Information System shows that the percentage of households with availability of soap and water increased from $43 \%$ to $57 \%$ from July to December 2020 to January to March 2021. ${ }^{17}$ The trained 446 CHWs who implemented household hygiene awareness activities at the community are part of the ecosystem that have contributed to this improvement. In the words of Community Health Worker Flora Mbuze: "The community acknowledges and recognizes our contributions. We are invited by local leaders to participate in decisions." 
A young mother from Kaliua District described how the community health worker explained COVID-19 to her: "I was not aware about this before, but now I am familiar with protecting myself and protecting others. In fact, the education he gave me helped me a lot and I applied some of the instructions he gave me. For example, in the beginning I used regular water for washing but the health worker instructed me to use running water and soap and I bought a special bucket with a watering can for handwashing."

Training participants have shown examples of ownership and leadership in GBV response. Following a training on GBV, an influential leader at the village level spoke about her willingness to engage with her committee on addressing GBV: "We are ready to send the message to offenders to stop violence immediately."

\section{DISCUSSION}

\section{LESSONS LEARNED}

\section{IMPLEMENT MULTI-SECTORAL INTEGRATED APPROACH}

Working with multiple government departments across health, social welfare and community development is key for a holistic, multi-sectoral approach. Core interventions for health system integration included infection prevention and control, integrated disease surveillance, gender response and community engagement to strengthen health systems resilience against infectious diseases including but not limited to COVID-19.

\section{BUILD ON EXISTING HEALTH SYSTEMS AND GOVERNMENT INITIATIVES FOR SUSTAINABILITY}

Building on existing government initiatives, guidelines, and management structures such as the regional and council/ district health management teams provides platforms for sustainability beyond the life of project and leveraging of government resources through partnership. Training government mentors at the regional and district levels on IPC provided a mechanism for ongoing mentorship and local capacity building. Supporting CHWs on awareness creation for household hygiene enabled ongoing community-level resilience to infectious disease threats.

\section{APPLY GENDER LENS IN CRISIS RESPONSE}

Engaging local organizations, existing community platforms, health care workers, and village committees in GBV response created a mechanism for local integrated health response to GBV and gender inequity accelerated by the pandemic. Leveraging existing community platforms through savings groups provided opportunities for community members to discuss and address gender dynamics and other social determinants influencing health behaviors.

\section{CONCLUSIONS}

In July 2021, President Hassan kicked off Tanzania's vaccination campaign with the receipt of 1.1 million doses of COVID-19 Johnson \& Johnson Vaccine from COVAX. With the recent rollout of COVID-19 vaccination in country, Tabora Region leveraged these robust health systems processes to ensure distribution, ongoing surveillance, and community engagement to support vaccine acceptance. In September 2021, Tabora updated its Regional COVID-19 response plan in response to National COVID-19 Response Plan. CARE Tanzania, along with other key development partners, supported the region through priority setting, activity prioritization, and rollout for COVID-19 vaccine distribution including community awareness and training of CHWs for community engagement.

Strengthening existing government health management structures from national to local levels across health, social welfare and community development departments allowed for integrated, holistic systems strengthening for response to disease threats including COVID-19. Leveraging existing community platforms provided opportunities for community members to discuss and address gender dynamics and other social determinants of health behaviors in their own lives and communities. Integrated approaches have potential for incremental health systems impacts across areas of holistic health and development across Sustainable Development Goals related to health, gender equality, and water and sanitation. ${ }^{8}$ These lessons are relevant for program managers in other contexts seeking to implement integrated, gender-responsive approaches to COVID-19 and other diseases. Program managers seeking to address COVID-19 in their contexts should strengthen health systems at regional, district, and community levels to enable resilient response to disease threats including but not limited to COVID-19.

\section{ACKNOWLEDGMENTS}

We thank Flavian Lihwa (Senior Program Manager, CARE Tanzania), Haika Mtui (Acting Country Director, CARE Tanzania), Nadia Lampkin (Program Officer, CARE Canada), and Allison Prather (Senior Technical Advisor, CARE USA) for their helpful review and comments to this manuscript. We thank the community members in Tabora Region who participated and continue to support the health system and community response to disease threats and GBV. We are grateful for the health care workers, community health workers, and regional/district government health management teams who partnered with us and continue to deliver critical services in challenging circumstances. Finally, we are grateful to the CARE Tanzania program staff who implemented these efforts hand in hand with community members and government counterparts. 


\section{FUNDING}

Program activities were funded by Bloomberg Philanthropies.

\section{AUTHORSHIP CONTRIBUTIONS}

SS conceptualized the paper. AI contributed to design and interpretation of data. SS led the manuscript drafting; AI edited all sections of the manuscript, contributed to manuscript sections, and provided key input. SS and AI approved the version to be published, and both are accountable for accuracy and integrity of the work.

\section{COMPETING INTERESTS}

The authors completed the Unified Competing Interest form at http://www.icmje.org/disclosure-of-interest/ (available upon request from the corresponding author) and declare no conflicts of interest.

\section{CORRESPONDENCE TO:}

Shegufta Shefa Sikder, PhD, MHS, 110017th St NW Suite 900, Washington, DC 20036, shefa.sikder@care.org

Submitted: December 06, 2021 GMT, Accepted: December 20, 2021 GMT 


\section{REFERENCES}

1. Tarimo CS, Wu J. The first confirmed case of COVID-19 in Tanzania: Recommendations based on lesson learned from China. Trop Med Health. 2020;48(1). doi:10.1186/s41182-020-00214-x

2. Tanzania COVID-19 Situation Report. World Health Organization Accessed August 1, 2021. https://covid1 9.who.int/region/afro/country/tz

3. British Broadcasting Corporation. Coronavirus: John Magufuli declares Tanzania free of Covid-19. Accessed August 1, 2021. https://www.bbc.com/news/ world-africa-52966016

4. Reuters. Tanzania leader launches COVID-19 vaccination drive, orders more jabs. Accessed August 1, 2021. https://www.reuters.com/world/africa/tanzan ia-leader-launches-covid-19-vaccination-drive-order s-more-jabs-2021-07-28/

5. Ministry of Health, Community Development, Gender, Elderly and Children, Ministry of Health, National Bureau of Statistics, Office of the Chief Government Statistician, ICF. Tanzania Demographic and Health Survey and Malaria Indicator Survey (TDHS-MIS) 2015-16. MoHCDGEC, NBS, OCGS, ICF; 2016. Accessed August 1, 2021. https://dhsprogram.c om/pubs/pdf/fr321/fr321.pdf

6. Cooperative for Relief and Assistance Everywhere, Inc. (CARE). Tabora Maternal \& Newborn Health Initiative (TAMANI): Year 4 Results. CARE; 2021. Accessed August 1, 2021. https://www.careevaluation s.org/evaluation/tabora-maternal-newborn-health-in itiative-tamani-year-4-results/

7. Nkowane BM. Streamlining and Strengthening the Disease Surveillance System in Tanzania.; 2019. Accessed August 1, 2021. https://www.mcsprogram.or $\mathrm{g} /$ resource/streamlining-and-strengthening-the-dise ase-surveillance-system-in-tanzania/

8. Cooperative for Relief and Assistance Everywhere, Inc. (CARE). CARE 2030 Vision. CARE; 2021. Accessed December 15, 2021. https://www.care-international.o rg/files/files/Vision_2030.pdf

9. Cooperative for Relief and Assistance Everywhere, Inc. (CARE). Rapid Gender Analysis Toolkit. Published online 2019. Accessed December 15, 2021. http://gender.careinternationalwikis.org/care_rapid_g ender_analysis_toolkit
10. Cooperative for Relief and Assistance Everywhere, Inc. (CARE). She Told Us So. CARE; 2020. Accessed December 15, 2021. https://www.care.org/wp-conten t/uploads/2020/09/RGA_SheToldUsSo_9.18.20.pdf

11. Mahuku E, Yihun KL, Deering K, Molosani B. CARE Rapid Gender Analysis for COVID 19 in East, Central and Southern Africa. CARE; 2020. Accessed August 1, 2021. https://careevaluations.org/wp-conte nt/uploads/ECSA-RGA- -FINAL-30042020.pdf

12. Government of United Republic of Tanzania, UNICEF. National Plan of Action to End Violence Against Women and Children in Tanzania 2017/ 8-2021/2. United Republic of Tanzania; 2016. Accessed August 1, 2021. https://www.unicef.org/tanz ania/reports/national-plan-action-end-violence-agai nst-women-and-children-tanzania-20178-20212

13. Ministry Of Health, Community Development, Gender, Elderly and Children. The National Guidelines for Water, Sanitation, and Hygiene in Health Care Facilities. United Republic of Tanzania; 2017.

Accessed August 1, 2021. https://washmatters.waterai d.org/sites/g/files/jkxoof256/files/National\%20Guideli nes\%20for\%20WASH\%20Services\%20in\%20Health\%2 0Care\%20Facilities-Tanzania.pdf

14. Ministry Of Health, Community Development, Gender, Elderly and Children, President's Office Regional Administration and Local Government. Comprehensive Council Health Plan Dissemination Training. United Republic of Tanzania; 2020. Accessed August 1, 2021. https://pubdocs.worldban k.org/en/842521595010580232/Tanzania-TF0A816 0-COMPILED-SLIDES-CCHP-DISSEMINATION-28-Fe bruary-2020-Final.pdf

15. Cooperative for Relief and Assistance Everywhere, Inc. (CARE). . Social Analysis and Action Global Implementation Manual. CARE; 2018. Accessed August 1, 2021. https://www.care.org/wp-content/upl oads/2020/08/saatoolkit_final_rights_reserved.pdf

16. United Nations Development Programme (UNDP). Rapid Socio-Economic Impact Assessment of COVID-19 in Tanzania. UNDP; 2020. Accessed August 1, 2021. ht tps://www.undp.org/content/dam/tanzania/docs/docs 2020/undp-tz-SEA-Report\%20Rapid-COVID19.pdf

17. Tanzania Water, Sanitation, and Hygiene Portal. Ministry of Health, Community Development, Gender, Elderly and Children website. Accessed August 1, 2021. https://nsmis.moh.go.tz/nsmisporta 1/\#/indicator 